\title{
TRAP1 and SORCIN cooperate in a survival pathway responsible for inducing drug-resistance in human colorectal carcinoma (CRC)
}

\author{
Matteo Landriscina ${ }^{1 *}$, Francesca Maddalena', Gabriella Laudiero², Maria R Amoroso², Annamaria Piscazzi ${ }^{1}$, \\ Valentina Lombardi ${ }^{1}$, Alberto Fersini ${ }^{3}$, Franca Esposito ${ }^{2}$
}

From 16th International Charles Heidelberger Symposium on Cancer Research

Coimbra, Portugal. 26-28 September 2010

TRAP1, a mitochondrial chaperone with antiapoptotic functions, is involved in drug-resistance in human CRC. TRAP1 is a component of the HSP90 mitochondrial survival pathway, which antagonizes the proapoptotic activity of cyclophilin D. Interestingly, novel agents are able to disassemble this pathway and are under clinical evaluation.

GST-pulldown experiments and mass spectrometry assays led us to the identification of several TRAP1 ligands and, among others, Sorcin, a $\mathrm{Ca}^{2+}$-binding protein involved in the MDR phenotype of human tumors. We functionally characterized the cooperation between TRAP1 and Sorcin in favoring survival of CRC cells and investigated the expression levels of the two proteins in human CRCs.

We demonstrated the mitochondrial localization of a $18 \mathrm{kDa}$ isoform of Sorcin that specifically interacts with TRAP1 in human CRC cells. The overexpression or down-regulation of Sorcin and/or TRAP1 allowed us to demonstrate a reciprocal regulation between the two proteins and to show that their interaction is required for Sorcin mitochondrial localization and TRAP1 protein stability. Furthermore, the upregulation of either TRAP1 and/or Sorcin induced resistance to 5-fluoruracil (FU), irinotecan (IRI) and oxaliplatin (l-OHP) in CRC cells. Of note, the knock-down of TRAP1 and/or Sorcin expression by siRNAs as well as the pharmacological inhibition of TRAP1 ATPase re-established a phenotype sensitive to FU, IRI and l-OHP. Consistently with these in vitro observations, gene expression analysis showed that TRAP1 and Sorcin are up-regulated, respectively, in 28/41 and 18/41 CRCs and a significant correlation between the expression of TRAP1 and the p22 isoform of Sorcin was observed (Sperman rank test $\mathrm{p}=0.003$; Kendal Tau test $\mathrm{p}=0.003$ ). Finally, the two proteins are also co-upregulated in CRC cells resistant to FU, IRI and l-OHP.

Sorcin and TRAP1 are functionally linked in a survival network responsible for resistance to chemotherapy in CRCs. Thus, TRAP1 pathway deserves to be further evaluated as molecular target to overcome drug resistance in human CRC.

\section{Author details \\ ${ }^{1}$ Clinical Oncology Unit, Department of Medical Sciences, University of Foggia, Foggia, Italy. ${ }^{2}$ Department of Biochemistry and Medical Biotechnology, University of Naples Federico II, Naples, Italy. ${ }^{3}$ General Surgery Unit, Department of Surgical Sciences, University of Foggia, Foggia, Italy.}

Published: 24 September 2010

\section{doi:}

Cite this article as: Landriscina et al.: TRAP1 and SORCIN cooperate in a survival pathway responsible for inducing drug-resistance in human colorectal carcinoma (CRC). BMC Proceedings 2010 4(Suppl 2):P37.

\footnotetext{
* Correspondence: m.landriscina@unifg.it

${ }^{1}$ Clinical Oncology Unit, Department of Medical Sciences, University of

Foggia, Foggia, Italy

Full list of author information is available at the end of the article
} 